Check for updates

Cite this: RSC Adv., 2019, 9, 8113

Received 26th December 2018 Accepted 6th March 2019

DOI: $10.1039 / c 8 r a 10574 j$

rsc.li/rsc-advances

\section{Facile synthesis of polyoxometalates tethered to post Fe-BTC frameworks for esterification of free fatty acids to biodiesel}

\author{
Qiuyun Zhang, $\mathbb{D} \dagger^{\star a c}$ Xiaofang Liu, $\dagger^{\mathrm{b}}$ Tingting Yang, ${ }^{a}$ Caiyan Yue, ${ }^{a}$ Quanlin $\mathrm{Pu}^{\mathrm{a}}$ \\ and Yutao Zhang ${ }^{\star a c}$
}

A phosphomolybdic acid (PMA) was sequentially incorporated into highly porous metal-organic frameworks (MOFs, Fe-BTC) by using a facile one-pot synthesis method, and the prepared composite (PMA/Fe-BTC) was employed as an efficient and stable solid acid catalyst for biodiesel production. $\mathrm{N}_{2}$ adsorption-desorption, X-ray diffraction (XRD), Fourier transform infrared spectroscopy (FT-IR), thermogravimetric (TG) analysis, scanning electron microscopy (SEM) and transmission electron microscopy (TEM) characterization techniques were employed to reveal the structure-performance relationship. The effects of reaction parameters like catalyst amount, reaction time and temperature were further investigated. This solid acid gave good catalytic performance in the esterification of free fatty acids with methanol, which is attributed to large surface area and thermal stability. The PMA/FeBTC was successfully reused for up to six cycles and exhibited high stability. From the kinetic study performed at different reaction temperatures $\left(140{ }^{\circ} \mathrm{C}, 150{ }^{\circ} \mathrm{C}\right.$ and $\left.160{ }^{\circ} \mathrm{C}\right) E_{a}=49.5 \mathrm{~kJ} \mathrm{~mol}^{-1}$ was obtained. In addition, due to the high activity presented in various esterifications of free fatty acids, this composite catalytic material has immense potential for industrial biodiesel production.

\section{Introduction}

Over the past decades, environmental and energy issues caused by excessive combustion of fossil fuels have aroused wide attention among researchers, and alternative renewable energy sources such as sunlight, wind and biofuels are encouraged to be developed., ${ }^{1,2}$ Among the renewable biofuels, biodiesel is considered a promising renewable source of energy alternative to fossil fuel due to its availability of feedstock, carbonneutrality, biodegradable, low emission of carbon dioxide, free of sulphur, possession of similar physical and chemical properties to those of petrodiesel and facilitating its direct usage in existing diesel engines with little or no modification. ${ }^{3}$ Generally, biodiesel (fatty acid methyl esters, FAMEs) is obtained from vegetable oils, ${ }^{4}$ animal fats ${ }^{5}$ and microalgal lipids ${ }^{6}$ by the process of esterification or transesterification. The current employed conventional method is no longer sustainable considering the high cost of food grade vegetable oils which

\footnotetext{
${ }^{a}$ School of Chemistry and Chemical Engineering, Anshun University, Anshun 561000, Guizhou, China. E-mail: sci_qyzhang@126.com

${ }^{b}$ Food and Pharmaceutical Engineering Institute, Guiyang University, Guiyang 550005, China

${ }^{c}$ Engineering Technology Center of Control and Remediation of Soil Contamination of Provincial Science \& Technology Bureau, Anshun University, Anshun, 561000, Guizhou, China.E-mail: zyt0516@126.com

$\dagger$ These authors contributed equally to this work.
}

account for more than $70 \%$ of the total cost of biodiesel production. ${ }^{7,8}$

More importantly, non-edible oils and waste cooking oils are more preferable as feedstocks for biodiesel production of commercial scale due to their low cost. However, non-edible oils and waste cooking oils usually contain large amount of free fatty acids (FFAs) are not suitable for base catalytic produce biodiesel because of serious saponification., ${ }^{9,10}$ Thus, great efforts directed towards the development of acidic catalysts have been made to catalyze esterification of FFAs. According to previous researches, ${ }^{\mathbf{1 1}}$ the esterification of long chain carboxylic acids such as oleic acid, lauric acid and palmitic acid can represent well the biodiesel production process, because this compound is present in most of the oil plants (rapeseed, soybean, sunflower, palm, Jatropha curcas, etc.). Unfortunately, liquid acids catalytic system such as $\mathrm{HCl}, \mathrm{H}_{2} \mathrm{SO}_{4}, \mathrm{H}_{3} \mathrm{PO}_{4}$ suffer problems of environmental pollution, equipment corrosion and inconvenient recycling. ${ }^{\mathbf{1 2 , 1 3}}$ These drawbacks could be overcome by using solid acid catalysts instead of liquid mineral acids. In the past few years, there has been exhaustive investigations focused on the search of solid acid catalysts for the esterification, including sulfonic acid-functionalized solid acid, ${ }^{\mathbf{1 4}}$ sulphated zirconia, ${ }^{15}$ heteropolyacids, ${ }^{16}$ metal oxides, ${ }^{17}$ zeolite, ${ }^{18}$ metal modified graphene oxide composite,${ }^{19}$ etc. But, these solid acid catalysts show that the disadvantages of poor mass transfer, low thermal stability and low acidity. 
The heteropolyacids (HPAs) were validated to be proper for esterification owing to the advantages of structural diversity, thermal stability, strong Brønsted acids and high oxidizing ability. ${ }^{20,21}$ However, HPAs also have some drawbacks, such as very high solubility in polar solvents and low specific surface area, which limit their application of the catalytic field..$^{22}$ In order to overcome these disadvantages, the exchange of protons of HPAs with different cations results in formation of insoluble salts and the immobilization of HPAs could be acted as efficient strategy for the catalytic reaction. Now, different supports have been explored to design HPA-immobilized solid acid catalysts for various organic transformations, including $\mathrm{SiO}_{2},{ }^{23}$ montmorillonite $\mathrm{K} 10,{ }^{24}$ mesoporous polymer of PDVB-VBC, ${ }^{25}$ or MCM-41. ${ }^{26}$ Unfortunately, the high solubility of HPAs in polar solvents and weak interaction between active HPAs and supported makes it relatively difficult to be immobilized on the above materials. Therefore, encapsulation of HPAs in cages and the pores of porous host framework can improve the interaction and avoid the leach of HPAs during different liquid medium operations.

Recently, metal-organic frameworks (MOFs) are a novel group of materials with controllable composition, designation, flexibility and easy preparation, a large number of uniformly sized micropores and large surface area. ${ }^{27,28}$ Various properties of MOFs have been applied to different fields, such as catalysis, gas adsorption, separation, electronic and optical properties. ${ }^{29-31}$ Due to the MOFs are highly designable, they have significant potential as a novel host materials for small guest molecules. Fujie, et al. ${ }^{32}$ reported an IL incorporated into the micropores of ZIF-8 and the phase behavior of the IL inside of the ZIF-8 micropores, and this result provides a novel strategy for stabilizing the liquid phase of the ILs down to a lower temperature region. Tharun, et al. ${ }^{33}$ prepared a novel material IL-ZIF-90 by the covalent postfunctionalization of n-ZIF-90 via grafting IL, and made use as a catalyst for the solvent less cycloaddition reaction to $\mathrm{CO}_{2}$ with various epoxides to provide cyclic carbonates, it shows excellent $\mathrm{CO}_{2}$ transforming capability.

In this work, we present a one-pot route to synthesis a series of HPAs tethered to post Fe-BTC frameworks composite as solid acid catalysts for a process of industrial and environmental relevance such as biodiesel production of oleic acid with methanol through esterification. The structures of the composite were analyzed by $\mathrm{N}_{2}$ adsorption-desorption, X-ray diffraction (XRD), scanning electron microscopy (SEM), transmission electron microscopy (TEM), Fourier transform infrared spectroscopy (FTIR), thermogravimetric (TG) analysis. Onward the effect of various process parameters have also been studied i.e., catalyst amount, FFAs to methanol molar ratio, reaction temperature and time. Meanwhile, the kinetic parameters of the reaction, along with catalyst reusability were discussed. Eventually, esterification of different free-fatty acids with methanol is also carried out to validate compatibility of the solid acid catalyst.

\section{Experimental}

\subsection{Materials}

Iron(III) nitrate nonahydrate $\left(\mathrm{Fe}\left(\mathrm{NO}_{3}\right)_{3} \cdot 9 \mathrm{H}_{2} \mathrm{O}\right.$, AR), phosphomolybdic acid $\left(\mathrm{H}_{3} \mathrm{PMO}_{12} \mathrm{O}_{40}\right.$, PMA, AR), 1,3,5-benzene tricarboxylic acid $\left(\mathrm{H}_{3}-\mathrm{BTC}\right)$ (AR), absolute ethanol (AR), oleic acid (AR), methanol (AR, >99\%), lauric acid (AR, 98\%), stearic acid (AR, 98\%), myristic acid (AR, 98\%), palmitic acid (AR, 98\%) and methanol (AR, >99\%), were purchased from Sinopharm Chemical Regent Co., Ltd. All other chemicals were of analytical grade and used as received, unless otherwise noted.

\subsection{Materials preparation and characterization}

The materials were prepared according to the literature method with slight modifications. ${ }^{34}$ A solution was prepared by dissolving $\mathrm{Fe}\left(\mathrm{NO}_{3}\right)_{3} \cdot 9 \mathrm{H}_{2} \mathrm{O}$ (3.0 mmol, $\left.1.21 \mathrm{~g}\right)$ and phosphomolybdic acid (PMA, $0.55 \mathrm{mmol}, 1.0 \mathrm{~g}$ ) in $12 \mathrm{~mL}$ of distilled water in a Teflon liner, and then $\mathrm{H}_{3}$-BTC $(3.0 \mathrm{mmol}, 0.63 \mathrm{~g}$ ) was added. After stirring at room temperature for $30 \mathrm{~min}$, the Teflon liner was sealed in a stainless-steel autoclave and maintained at $120{ }^{\circ} \mathrm{C}$ for $6 \mathrm{~h}$. After the heat treatment, the autoclave cool naturally to room temperature, the resultant solid was isolated by centrifugation and then washed in $150 \mathrm{~mL}$ of ethanol to remove unreacted $\mathrm{H}_{3}$-BTC. Then, the powder was then treated with boiling ethanol for $3 \mathrm{~h}$ several times. Follow, the obtained composite was centrifuged and dried in an oven at $60{ }^{\circ} \mathrm{C}$ for $24 \mathrm{~h}$ (denoted as PMA/Fe-BTC). For comparison, Fe-BTC sample without PMA was also synthesized under the same conditions and denoted as Fe-BTC. The obtained catalysts were dried at $120{ }^{\circ} \mathrm{C}$ before use for the catalytic esterification reaction. The XRD patterns of the catalysts were obtained using a Rigaku D/ max 2000 ultima plus diffractometer (monochromatic nickel filter, $\mathrm{Cu} \mathrm{K} \alpha$ radiation). The FT-IR spectra was scanned on a PerkinElmer spectrum 100 using the $\mathrm{KBr}$ disc technique (4000-400 $\mathrm{cm}^{-1}$ ). Thermogravimetric (TG) analysis was performed on NETZSCH/STA 409 PC Luxx simultaneous thermal analyzer with a heating rate of $5{ }^{\circ} \mathrm{C} \mathrm{min}^{-1}$ under an air flow rate of $20 \mathrm{~mL} \mathrm{~min}{ }^{-1}$. The BET surface area, total pore volume and pore size distribution of the catalyst were determined with Micromeritics ASAP 2020 V4.02 volumetric adsorption analyzer. Scanning electron microscopy (SEM) images were obtained on JEOL-6701F scanning electron microscope at $10.0 \mathrm{kV}$. Transmission electron microscopy (TEM) was performed on a JEOL $2100 \mathrm{~F}$ electron microscope operated at $200 \mathrm{kV}$.

\subsection{The esterification test}

The catalytic properties of PMA/Fe-BTC were measured by esterification of oleic acid with methanol. Oleic acid (about 2.82 $\mathrm{g})$, dehydrated methanol and catalyst were reacted in a stainless steel high-pressure autoclave reactor containing a magnetic stirrer and temperature controller by oil bath system, and the resulting mixture was heated at $160{ }^{\circ} \mathrm{C}$ for $3 \mathrm{~h}$ under autogenous pressure (1.0-1.2 $\mathrm{MPa}$ ). After the reaction, the catalysts were separated by centrifugation and washed with methanol. Then, catalyst was directly used for next run. Besides, the reaction products were purified under reduced pressure to remove the excess methanol, and were examined by titration with $0.1 \mathrm{~N}$ $\mathrm{KOH}$ using phenolphthalein indicator to determine the acid value (AV) by following formulas referring to ISO 660-2009 standard. From the acid value, the conversion to oleic acid can be obtained by the following equation. 


$$
\text { Conversion }(\%)=\frac{\mathrm{AV}_{1}-\mathrm{AV}_{2}}{\mathrm{AV}_{1}} \times 100 \%
$$

$\mathrm{AV}_{1}$ - acid value of raw materials; $\mathrm{AV}_{2}$ - acid value of esterification product.

\section{Results and discussion}

\subsection{Characterization}

3.1.1. XRD analysis of catalyst. X-ray diffractometer (XRD) of the pristine PMA, Fe-BTC and PMA/Fe-BTC catalyst is given in Fig. 1. Pristine PMA showed seven main diffraction peaks at $7.6^{\circ}, 25.9^{\circ}$ and $28.0^{\circ}$, which can be attributed to the structure of Keggin anion. For the Fe-BTC sample, the spectra are in good agreement with previous literature reports ${ }^{35}$ but the prepared material demonstrated low crystallinity. With the introduction to the PMA species, some diffraction peaks corresponding to PMA were observed in the PMA/Fe-BTC sample and no noticeable changes in the crystal structure, probably suggesting that the PMA clusters are mainly encaged within the Fe-BTC cavities. Noteworthy, the intensity of diffraction peaks corresponding to PMA were observed in the PMA/Fe-BTC are obvious higher than these in pristine PMA, which suggests that there may exist few PMA crystals on the outer surface of Fe-BTC. Based on above analysis, this suggest the successful synthesis tethered post FeBTC materials.

3.1.2. FT-IR analysis of the catalyst. The FT-IR spectrum of pristine PMA, Fe-BTC and PMA/Fe-BTC samples are displayed in Fig. 2. For Fe-BTC and PMA/Fe-BTC, the peaks at approximately $1718 \mathrm{~cm}^{-1}, 1636 \mathrm{~cm}^{-1}, 1585 \mathrm{~cm}^{-1}, 1454 \mathrm{~cm}^{-1}$ and $1383 \mathrm{~cm}^{-1}$, corresponding to the presence of -COOFe metallic esters in this compound. Among them, two characteristic bands of $1636 \mathrm{~cm}^{-1}$ and $1585 \mathrm{~cm}^{-1}$ were attributed to the splitting of the coordinated $-\mathrm{CO}^{2-}$ vibrations, $1718 \mathrm{~cm}^{-1}$ was attributed to $\mathrm{C}=\mathrm{O}$ stretching mode. In addition, the characteristic bands of $759 \mathrm{~cm}^{-1}$ and $710 \mathrm{~cm}^{-1}$ can be assigned to the existence of $\mathrm{C}-$ $\mathrm{CO}_{2}$ bond in the framework material, our results came close to previously reported results. ${ }^{36}$ When the PMA is encaged onto the Fe-BTC framework, pristine PMA and PMA/Fe-BTC also contain such bands. In the case of pristine PMA, the strong absorption

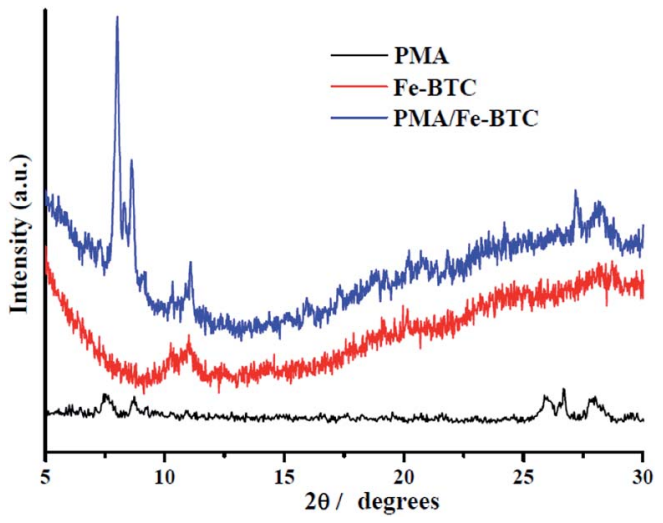

Fig. 1 XRD patterns of pristine PMA, Fe-BTC and PMA/Fe-BTC catalyst.

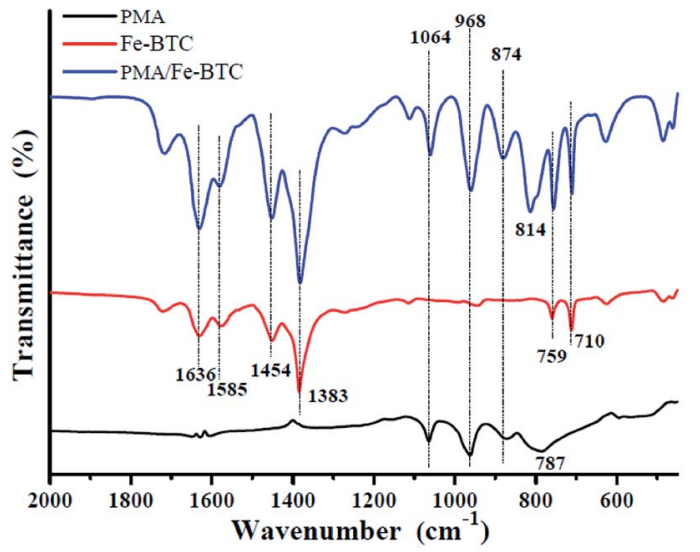

Fig. 2 FT-IR spectra of pristine PMA, Fe-BTC and PMA/Fe-BTC catalyst.

of PMA with Keggin structure band of $1064 \mathrm{~cm}^{-1}, 968 \mathrm{~cm}^{-1}$, $874 \mathrm{~cm}^{-1}$ and $787 \mathrm{~cm}^{-1}$ can be assigned to the $\mathrm{P}-\mathrm{O}, \mathrm{Mo}=\mathrm{O}$, Mo-Oc-Mo and Mo-Oe-Mo asymmetric stretching vibrations, respectively. ${ }^{37}$ Comparing with that of pristine PMA, PMA/FeBTC exhibits similar four characteristic vibration peaks at $1064 \mathrm{~cm}^{-1}, 968 \mathrm{~cm}^{-1}, 874 \mathrm{~cm}^{-1}$ and $814 \mathrm{~cm}^{-1}$. All the results suggest that PMA has been successfully encaged into the cavities of Fe-BTC and the classical Keggin structures were retained.

3.1.3. TG analysis of the catalyst. Thermal stability is of significant importance of materials used as ingredients of solid catalysts. PMA/Fe-BTC sample was subjected to thermogravimetry (TG) analysis to evaluate its thermal stability as shown in Fig. 3. As shown in Fig. 3, the $15.5 \%$ loss in the range of $40{ }^{\circ} \mathrm{C}$ to $155{ }^{\circ} \mathrm{C}$ can be assigned to the loss of water molecules. The second weight loss (5.7\%) ranging from $155{ }^{\circ} \mathrm{C}$ to $400{ }^{\circ} \mathrm{C}$ is due to the thermal decomposition of constitutional water $\left(\mathrm{H}_{3} \mathrm{O}^{+}\right)$ from PMA. Remarkably, further weight loss $(25.0 \%)$ from $400{ }^{\circ} \mathrm{C}$ to $700{ }^{\circ} \mathrm{C}$ was mainly ascribed to the thermal decomposition of organic BTC ligands. On the basis of these results, it can be demonstrated that the PMA/Fe-BTC material was highly thermal stable below $400{ }^{\circ} \mathrm{C}$ and the incorporation of PMA had no significant effect on its thermal stability, and has immense potential for esterification reaction of biodiesel production.

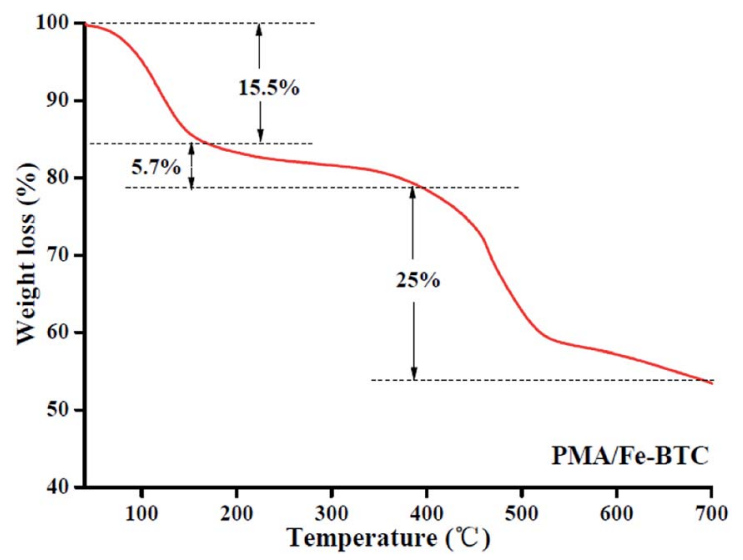

Fig. 3 TG analysis of PMA/Fe-BTC catalyst. 
3.1.4. BET analysis of the catalyst. Detailed textural properties of the PMA/Fe-BTC catalyst was determined from $\mathrm{N}_{2}$ sorption isotherms. The results showed the PMA/Fe-BTC catalyst possessed large measured surface area $\left(108.3 \mathrm{~m}^{2} \mathrm{~g}^{-1}\right)$ and an average pore diameter of $2.9 \mathrm{~nm}$ with a narrow pore size distribution.

3.1.5. SEM and TEM analysis of the catalyst. SEM was then applied to investigate the morphological properties of pristine PMA, and Fe-BTC, PMA/Fe-BTC (see Fig. 4a-c). As shown in Fig. 4a and b, the SEM micrograph of pristine PMA showed some agglomerates with undefined shapes and mixture of larger, and the Fe-BTC showed nearly spherical shapes in the aggregates of the irregular intergrown crystallites. After encapsulation process of the PMA (see Fig. 4c), the surface of PMA/Fe-BTC becomes uneven shapes and more coalesced, and cannot be distinguished from each other. The characterization results suggested that the pristine PMA encapsulated onto the surface of Fe-BTC. In addition, TEM was also employed to elucidate the morphologies of the synthesized PMA/Fe-BTC catalyst (see Fig. 4d). As observed, PMA/Fe-BTC displays an irregularity shape with a rough surface, which was consistent with SEM analysis. The SEM and TEM provided proof of the successful preparation of PMA tethered to post Fe-BTC frameworks.

\subsection{Esterification of oleic acid with methanol}

3.2.1. Effect of oleic acid to methanol molar ratio. As we all know, the esterification reaction could be accelerated by increasing the amount of methanol. Therefore, we try to further explore the effects of the oleic acid to methanol molar ratio for PMA/Fe-BTC catalyst, the esterification experiments were conducted by changing the molar ratio from $1: 4$ to $1: 24$, while keeping the temperature, time and the catalyst loading constant at $160{ }^{\circ} \mathrm{C}, 3 \mathrm{~h}$ and $10 \mathrm{wt} \%$, respectively. As shown in Fig. 5a, when the molar ratio increased from $1: 4$ to $1: 16$, the
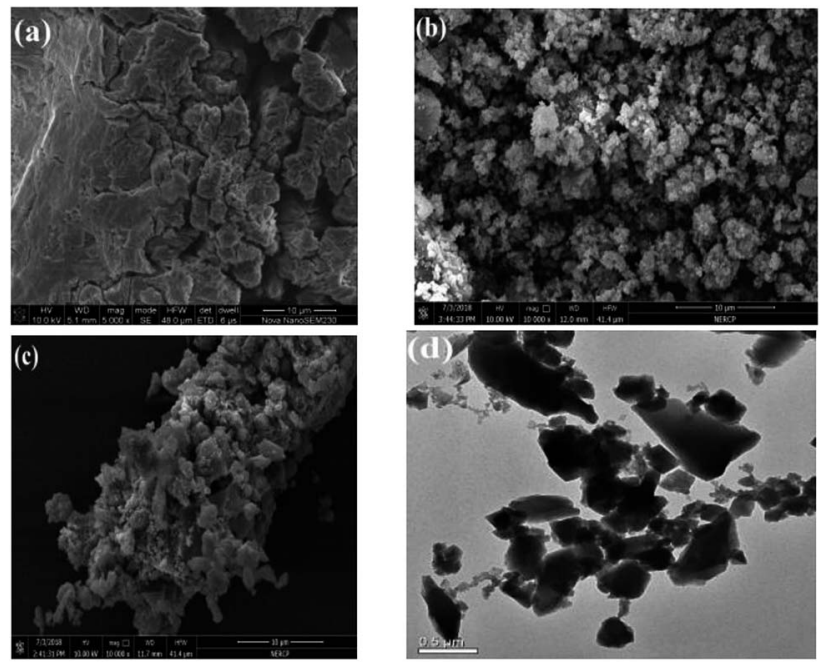

Fig. 4 SEM images of pristine PMA (a), Fe-BTC (b), PMA/Fe-BTC (c) catalyst and TEM images of the PMA/Fe-BTC (d) catalyst.
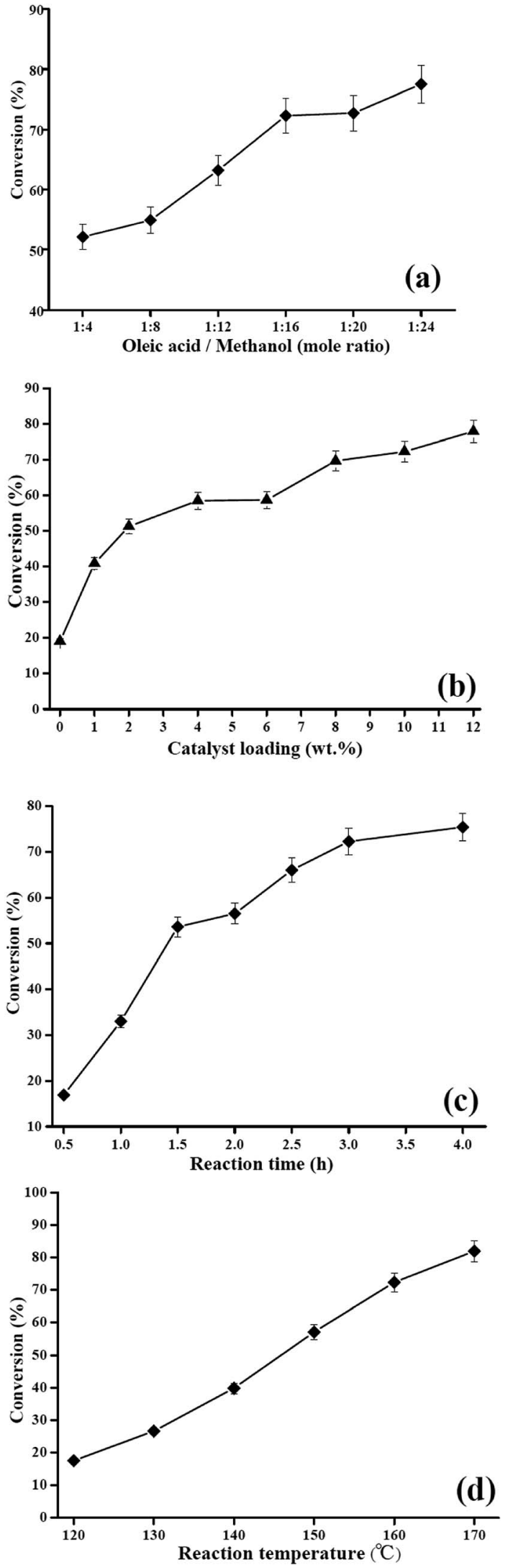

Fig. 5 The esterification of oleic acid by catalyst PMA/Fe-BTC at different reaction conditions (effects of molar ratio (a), catalyst amount (b), time (c) and temperature (d) on the oleic acid esterification conversion). 
conversion of methyl oleate rose significantly from $52.1 \%$ to $72.3 \%$ after the reaction for $3 \mathrm{~h}$. However, it was observed that altering the oleic acid/methanol molar ratio in the $1: 20$ to $1: 24$ ratio range had no significant effect on the conversion. Thus, a molar ratio $1: 16$ of oleic acid to methanol was observed to be the optimum.

3.2.2. Effect of catalyst loading. The catalyst loading was also an important parameter affecting the esterification of biodiesel production. Fig. 5b illustrates the change of esterification conversion as a function of catalyst loading with oleic acid to methanol molar ratio $1: 16$, reaction temperature $160{ }^{\circ} \mathrm{C}$ and reaction time $3 \mathrm{~h}$. The mass ratio of the PMA/Fe-BTC catalyst to oleic acid was varied within the range of 1-12 wt\%. When the catalyst loading increased from 1 to $10 \mathrm{wt} \%$, esterification conversion was raised from $40.9 \%$ to $72.3 \%$, this is due to the availability of active species. Above $10 \mathrm{wt} \%$ catalyst loading, a marginal rise in the conversion of methyl oleate was observed. Depending on the results, it can be deduced that $10 \mathrm{wt} \%$ catalyst loading of PMA/Fe-BTC can provide enough active acidic sites for the esterification, and the excessive acidic sites are not necessary. Similar observations were reported earlier. ${ }^{38}$ Hence, further reactions were performed at the optimum catalyst loading of $10 \mathrm{wt} \%$.

3.2.3. Effect of reaction time. 2To further evaluate the catalytic performance of PMA/Fe-BTC, its catalytic activity was tested at various reaction time $(0.5-4 \mathrm{~h})$ under constant other reaction conditions: oleic acid to methanol molar ratio of $1: 16$, reaction temperature $160{ }^{\circ} \mathrm{C}$ and catalyst loading of $10 \mathrm{wt} \%$. As shown in Fig. 5c, an oleic acid conversion of $16.9 \%$ was reached after a short reaction time $(0.5 \mathrm{~h})$. This can be attributed to slow mass transfer, which caused poor mixing and dispersion of the solid reactant. ${ }^{39}$ However, the increase in the reaction time enhanced the oleic acid conversion, the conversion of methyl oleate increased from $33.0 \%$ at $1 \mathrm{~h}$ and peaked to $72.3 \%$ at $3 \mathrm{~h}$. With a further prolongation of reaction time beyond $3 \mathrm{~h}$, the oleic acid conversion was slightly increased to $75.4 \%$ due to the reverse esterification process. ${ }^{40}$ Therefore, the reaction time of $3 \mathrm{~h}$ was chosen as the optimum condition and used for further investigation in the present research.

3.2.4. Effect of reaction temperature. Reaction temperature is another important parameter that influences the esterification rate because the intrinsic rate constants are strong functions of temperature. ${ }^{41}$ The esterification conversion process was carried out at $120,130,140,150,160$ and $170{ }^{\circ} \mathrm{C}$ with $10 \mathrm{wt} \%$ catalyst and an oleic acid to methanol molar ratio of $1: 16$ for $3 \mathrm{~h}$. Fig. $5 \mathrm{~d}$ showed that the esterification conversion process was positively affected by the increasing temperature, the oleic acid conversion increased from $17.5 \%$ to $72.3 \%$ as the reaction temperature changed from $120{ }^{\circ} \mathrm{C}$ to $160{ }^{\circ} \mathrm{C}$. With further increase in the reaction temperature a non-significantly increased in conversion was observed. Considering energy consumption, $160{ }^{\circ} \mathrm{C}$ as chosen as the optimal reaction temperature.

\subsection{PMA/Fe-BTC catalyst reusability}

Catalyst recyclability is an essential step for commercial feasibility since it reduces the biodiesel production budget. ${ }^{42}$ To examine recyclability of PMA/Fe-BTC catalyst, six consecutive runs of esterification of oleic acid with methanol were conducted using recovered PMA/Fe-BTC without any treatment (see Fig. 6). It is surprising that the catalyst showed high operational stability even after six recycling experiments retaining $59.2 \%$ of its original esterification activity. Partial loss in activity was probably due to leaching of PMA is directly related to their activities and the blockage of the energetic centers as a result of the accumulation of intermediates, oleic acid and products in the pore of the catalyst. Therefore, these results clearly demonstrate excellent recyclability of PMA/Fe-BTC.

\subsection{Oleic acid esterification reaction kinetics over PMA/Fe- BTC}

Such phenomena can also be confirmed with a kinetic analysis. Therefore, in this study, a detail study on kinetic behaviour for esterification of oleic acid with methanol was carried out over PMA/Fe-BTC at the optimum conditions of $10 \mathrm{wt} \%$ catalyst loading, oleic acid to methanol molar ratio of $1: 16$ for $3 \mathrm{~h}$, and the reaction and apparent activation energies was also calculated. Firstly, the PMA/Fe-BTC catalyst at $140{ }^{\circ} \mathrm{C}, 150{ }^{\circ} \mathrm{C}, 160{ }^{\circ} \mathrm{C}$ were used in esterification and the results are shown in Fig. 7a. It was noted that the different temperature significantly affected the conversion of oleic acid.

Here, we simplified the mathematical model for this reaction as follows:

$a$ Oleic acid (A) $+b$ methanol $(\mathrm{B}) \rightleftharpoons c$ methyl oleate $(\mathrm{C})$

$$
+d \text { water (D) }
$$

The reaction rate $(r)$ according to the following equation is:

$$
r=-\frac{\mathrm{d} C_{\mathrm{A}}}{\mathrm{d} t}=k^{\prime} C_{\mathrm{A}}^{a}{ }^{a} C_{\mathrm{B}}{ }^{b}-k^{\prime \prime} C_{\mathrm{C}}{ }^{c} C_{\mathrm{D}}{ }^{d}
$$

where $C_{\mathrm{A}}, C_{\mathrm{B}}, C_{\mathrm{C}}$, and $C_{\mathrm{D}}$ were the concentrations of oleic acid, methanol, methyl oleate, and water, respectively. $a, b, c$, and $d$ refer to their reaction order. $k^{\prime}$ and $k^{\prime \prime}$ are the kinetic constants for the forward and backward reactions, respectively. However, the concentration of methanol was not considered, since it was

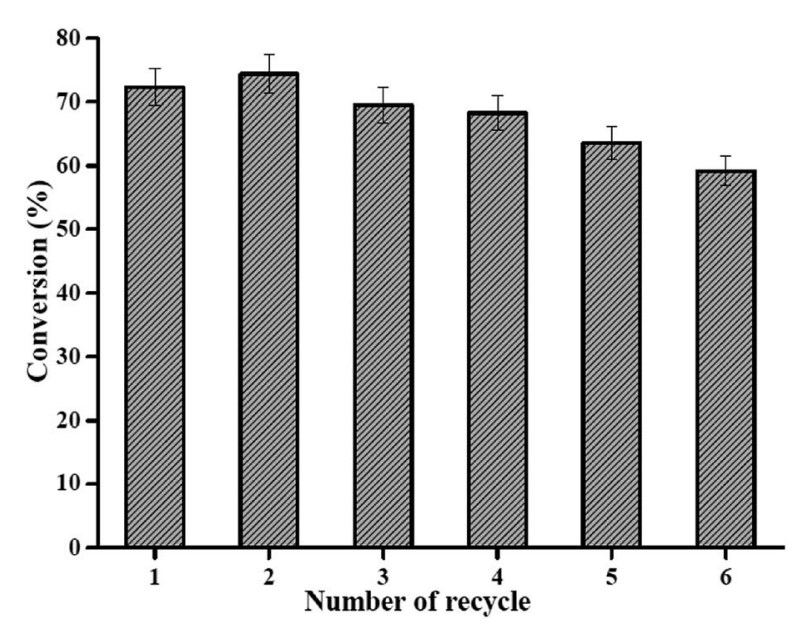

Fig. 6 Effect of catalyst reusability on the esterification reaction. 

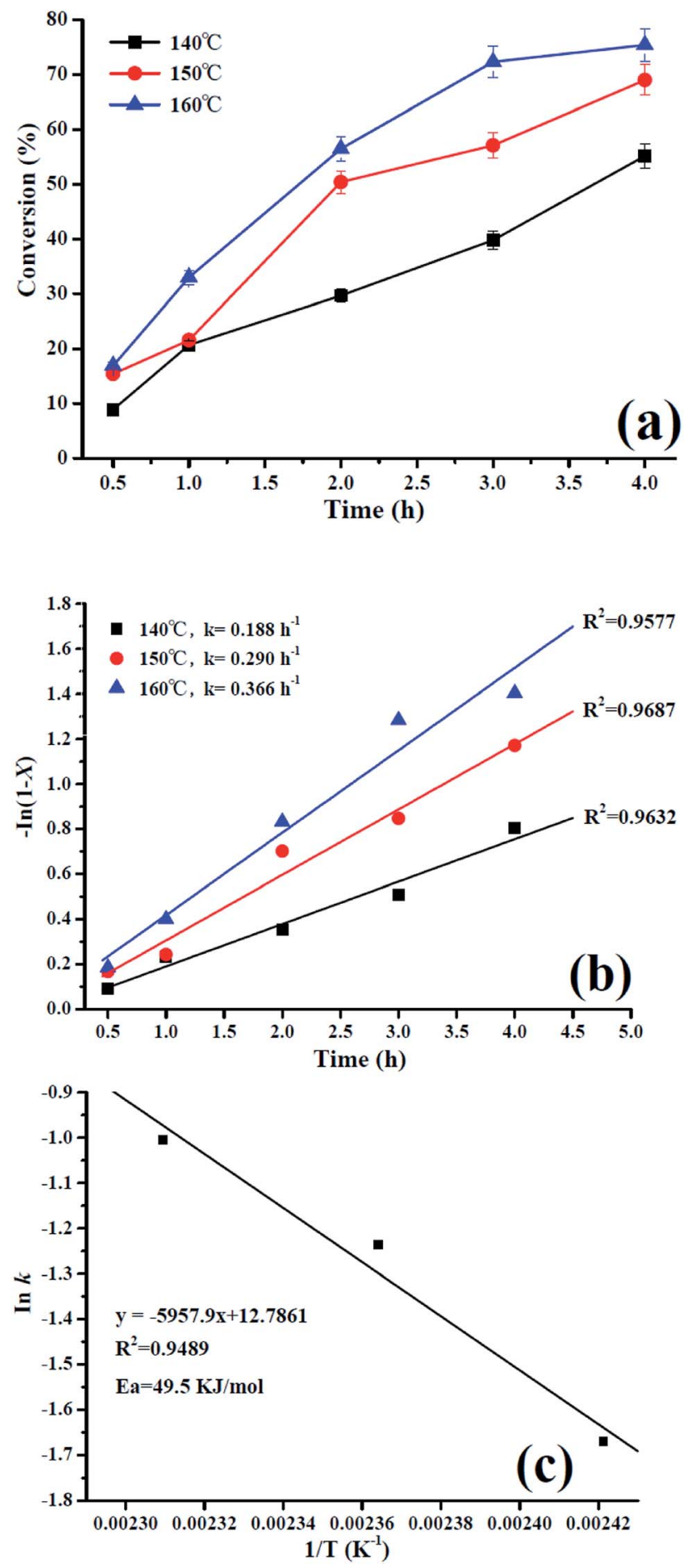

Fig. 7 Esterification reaction kinetics at different temperatures: (a) conversion with time at different temperatures; (b) linear relationship between $-\ln (1-X)$ and time at different reaction temperatures; (c) Arrhenius plot of $\ln k$ versus $1 / T$.

used in excess and its concentration can be considered unchanged throughout the process. Thus, $k^{\prime} \mathrm{C}_{\mathbf{B}}{ }^{b}$ can be constant. In addition, $k^{\prime}$ is much larger than $k^{\prime \prime}$. As the reaction rate depends only on the oleic acid concentration, the reaction was considered to be of first order. ${ }^{\mathbf{4 3 4 4}}$ Therefore, the law of the first order velocity is written by the equation:

$$
-\frac{\mathrm{d} C_{\mathrm{A}}}{\mathrm{d} t}=k C_{\mathrm{A}}
$$

where $k$ is modified rate constant and $k=k^{\prime} \mathrm{C}_{\mathrm{B}}{ }^{b}$.

$$
C_{\mathrm{A}}=C_{\mathrm{A} 0}(1-X)
$$

where $X$ and $C_{\mathrm{A} 0}$ are conversion of used oleic acid and the initial concentration of oleic acid, respectively. Thus, the above equation can be rearranged and integrated providing eqn (6):

$$
-\ln (1-X)=k t
$$

According to eqn (6), plots of $-\ln (1-X) v s$. time were made for different temperatures and is shown in Fig. 7b. In addition, the activation energy of the esterification of oleic acid in methyl oleate can be determined by the Arrhenius equation:

$$
\ln k=-E_{\mathrm{a}} / R T+\ln A
$$

From the Arrhenius plot (see Fig. 7c), the activation energy value for the reaction in the presence of the PMA/Fe-BTC catalyst was $49.5 \mathrm{~kJ} \mathrm{~mol}^{-1}$, and the activation energy lower than the previously reported values (e.g. $52.3 \mathrm{~kJ} \mathrm{~mol}^{-1}, 78 \mathrm{~kJ} \mathrm{~mol}^{-1}$, etc.). ${ }^{\mathbf{4 5 , 4 6}}$ Moreover, according to previous reported, ${ }^{47,48}$ the esterification reaction is not diffusively controlled but kinetically controlled if the apparent activation energy is high enough (e.g., $>20.0 \mathrm{~kJ} \mathrm{~mol}^{-1}$ ), and that the high apparent activation energy of esterification catalyzed by the PMA/Fe-BTC catalyst further demonstrates that diffusion is not important during our esterification processes. This finding suggests that the esterification reaction is chemically controlled and not by diffusion or mass transfer limitations.

\subsection{Catalytic performance of PMA/Fe-BTC for other esterifications}

To examine the scope of PMA/Fe-BTC catalyst in biodiesel production, further studies esterifications of other FFAs with methanol were studied. For all the fatty acids examined, the high conversions were achieved within $3 \mathrm{~h}$, e.g. $84.3 \%$ of lauric acid, $82.1 \%$ of myristic acid, $66.7 \%$ of palmitic acid and $76.0 \%$ of stearic acid. Based on the results, the PMA/Fe-BTC catalyst can effectively convert FFAs of various chain lengths of their methyl esters, which is the key pre-treatment step to remove FFA from low-cost non-edible oils and meet the demand of synthetic biodiesel.

\subsection{Catalytic activities of different catalysts}

Catalytic performance in esterification from FFAs over various catalysts is shown in Table 1 . As shown in Table 1 , the PMA/FeBTC exhibits relatively high catalytic activity with $72.3 \%$ conversion under mild reaction conditions, whereas the other catalyst is subjected to more or less drawbacks of relatively low conversion $(40 \%)$, require high temperature $\left(200{ }^{\circ} \mathrm{C}\right)$ and relatively long reaction time $(5 \mathrm{~h})$. Above analysis indicated that the PMA/Fe-BTC catalyst could be the promising catalysts for esterification reaction. 
Table 1 Comparison of performance of several catalysts with the present work for esterification

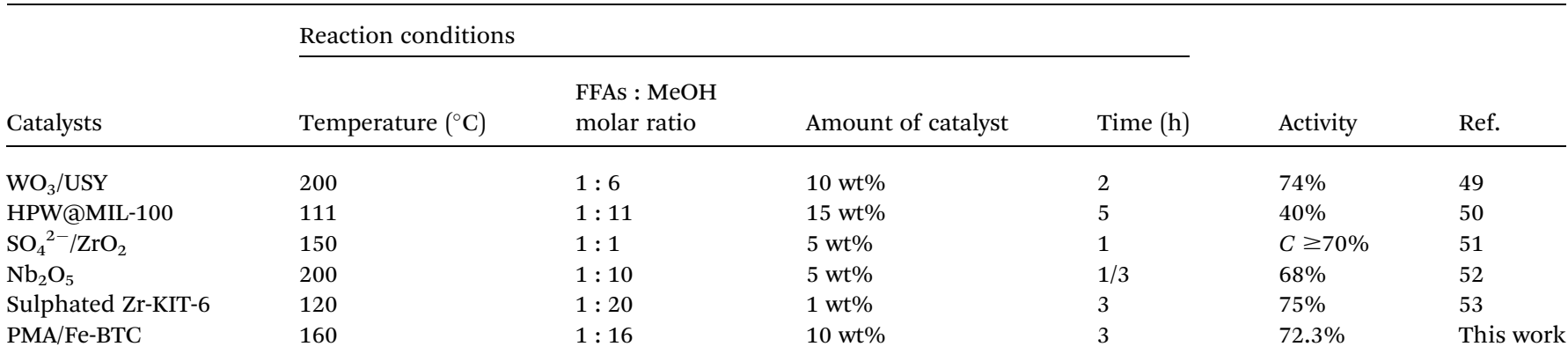

\section{Conclusions}

In the present study, we have successfully fabricated PMA/FeBTC composite as an efficient and stable catalyst by one-pot method that suitable for biodiesel production. The PMA/FeBTC gave high catalytic activity in the esterification, and could be attributed to filled active species (PMA), large surface area, thermal stability and the good protection measures of dual function porous material supports. Under the condition of catalyst loading $10 \mathrm{wt} \%$, reaction temperature $160{ }^{\circ} \mathrm{C}$ and oleic acid to methanol molar ratio of $1: 16$, the conversion rate reached $72.3 \%$ only in $3 \mathrm{~h}$. The kinetic studies reveal that the esterification can present a pseudo first-order kinetic process and the apparent activation energy is $49.5 \mathrm{~kJ} \mathrm{~mol}^{-1}$. Moreover, PMA/Fe-BTC showed an excellent stability in use. Hopefully, this green, simple and efficient method for the production of PMA/Fe-BTC catalytic composite used in esterification process would have significantly potential application for industrial biodiesel production.

\section{Conflicts of interest}

There are no conflicts to declare.

\section{Acknowledgements}

This work was financially supported by the Technical Talent Support Program of Guizhou Education Department (No. KY [2018]069 and KY [2017]093), the Joint Science and Technology Funds of Guizhou S\&T Department, Anshun City People's Government and Anshun University (No. LH [2016]7278), the Creative Research Groups Support Program of Guizhou Education Department (No. KY [2017]049), Construction Projects of Innovation Platform from Science and Technology Bureau in Anshun (No. [2016]-4), Guizhou Science \& Technology Foundation (No. [2019]1009), the Youth Growth S\&T Personnel Foundation of Guizhou Education Department (KY [2018] 292), the Start-up Funding Research Projects of Guiyang University Introduced Talents (No. GYU-ZRD [2018]-015).

\section{Notes and references}

1 N. Mansir, S. H. Teo, U. Rashid, M. I. Saiman, Y. P. Tan, G. A. Alsultan and Y. H. Taufiq-Yap, A review, Renewable Sustainable Energy Rev., 2018, 82, 3645-3655.
2 S. K. Yellapu, K. R. Bharti, L. R. Kumar, B. Tiwari, X. L. Zhang and R. D. Tyagi, Bioresour. Technol., 2018, 256, 515-528.

3 M. Anwar, M. G. Rasul and N. Ashwath, Energy Convers. Manage., 2018, 156, 103-112.

4 M. Adnan, K. Li, L. Xu and Y. J. Yan, Catalysts, 2018, 8, 96110.

5 L. Šánek, J. Pecha, K. Kolomazník and M. Bařinová, Waste Management, 2016, 48, 630-637.

6 X. Meng, J. Yang, X. Xu, L. Zhang, Q. Nie and M. Xian, Renewable Energy, 2009, 34, 1-5.

7 Q. Y. Zhang, F. F. Wei, P. H. Ma, Y. T. Zhang, F. H. Wei and H. L. Chen, Waste Biomass Valorization, 2018, 9, 911-918.

8 L. Li, P. W. Dyer and G. H. Christopher, ACS Omega, 2018, 3, 6804-6811.

9 Y. Pan, M. A. Alam, Z. M. Wang, J. C. Wu, Y. Zhang and Z. H. Yuan, Bioresour. Technol., 2016, 220, 543-548.

10 H. Zhang, H. Li, H. Pan, A. P. Wang, S. Souzanchi, C. B. Xu and S. Yang, Appl. Energy, 2018, 223, 416-429.

11 K. Y. Nandiwale and V. V. Bokade, Ind. Eng. Chem. Res., 2014, 53, 18690-18698.

12 Q. Y. Zhang, H. Li, X. F. Liu, W. T. Qin, Y. P. Zhang, W. Xue and S. Yang, Energy Technol., 2013, 1, 735-742.

13 Q. Y. Zhang, F. F. Wei, Y. T. Zhang, F. H. Wei, P. H. Ma, W. Zheng, Y. T. Zhao and H. L. Chen, J. Oleo Sci., 2017, 66, 491-497.

14 M. L. Tao, H. Y. Guan, X. H. Wang, Y. C. Liu and R. F. Louh, Fuel Process. Technol., 2015, 138, 355-360.

15 B. Banerjee, S. Bhunia and A. Bhaumik, Appl. Catal., A, 2015, 502, 380-387.

16 Q. Y. Zhang, F. F. Wei, Q. Li, J. S. Huang, Y. M. Feng and Y. T. Zhang, RSC Adv., 2017, 7, 51090-51095.

17 Q. Y. Zhang, H. Li and S. Yang, J. Oleo Sci., 2018, 67, 579-588. 18 A. Pacuła, K. Pamin, J. Kryściak-Czerwenka, Z. Olejniczak, B. Gil, E. Bielánska, R. Dula, E. M. Serwicka and A. Drelinkiewicz, Appl. Catal., A, 2015, 498, 192-204.

19 T. M. M. Marso, C. S. Kalpage and M. Y. UdugalaGanehenege, Fuel, 2017, 199, 47-64.

20 K. L. Yang, S. Huang, H. Pan, H. Zhang, X. F. Liu and S. Yang, RSC Adv., 2017, 7, 33335-33343.

21 L. J. Liu, Q. J. Luan, J. Lu, D. M. Lv, W. Z. Duan, X. Wang and S. W. Gong, RSC Adv., 2018, 8, 26180-26187.

22 Z. Zhao and X. Wang, Appl. Catal., A, 2016, 526, 139-146.

23 A. A. Elmekawy, N. R. Shiju, G. Rothenberg and D. R. Brown, Ind. Eng. Chem. Res., 2014, 53, 18722-18728. 
24 B. Vijayakumar and G. R. Rao, J. Porous Mater., 2012, 19, 233242.

25 S. P. Fu, J. F. Chu, X. Chen, W. F. Li and Y. F. Song, Ind. Eng. Chem. Res., 2015, 54, 11534-11542.

26 S. Singh and A. Patel, Fuel, 2015, 159, 720-727.

27 L. M. Huang, H. T. Wang, J. X. Chen, Z. B. Wang, J. Y. Sun, D. Y. Zhao and Y. S. Yan, Microporous Mesoporous Mater., 2003, 58, 105-114.

28 H. Q. Zheng, Y. N. Zhang, L. F. Liu, W. Wan, F. Guo, A. M. Nyström and X. D. Zou, J. Am. Chem. Soc., 2015, 138, 962-968.

29 H. L. Li, M. Eddaoudi, M. O'Keeffe and O. M. Yaghi, Nature, 1999, 402, 276-279.

30 K. Shen, L. Zhang, X. Chen, L. Liu, D. Zhang, Y. Han, J. Chen, J. Long, R. Luque, Y. Li and B. Chen, Science, 2018, 359, 206210.

31 C. S. Diercks, Y. Liu, K. E. Cordova and O. M. Yaghi, Nat. Mater., 2018, 17, 301-307.

32 K. Fujie, T. Yamada, R. Ikeda and H. Kitagawa, Angew. Chem., Int. Ed., 2015, 126, 11484-11487.

33 J. Tharun, K. M. Bhin, R. K. Roshan, D. W. Kim, A. C. Kathalikkattil, R. Babu, H. Y. Ahn, Y. S. Won and D. W. Park, Green Chem., 2016, 18, 2479-2487.

34 L. H. Wee, S. R. Bajpe, N. Janssens, I. Hermans, K. Houthoofd, C. E. A. Kirschhock and J. A. Martens, Chem. Commun., 2009, 46, 8186-8188.

35 X. P. Yang, X. X. Guo, C. H. Zhang, Y. Yang and Y. W. Li, Chin. J. Mater. Res., 2017, 31, 569-575.

36 Y. J. Yang, Y. Bai, F. Q. Zhao, E. G. Yao, J. H. Yi, C. L. Xuan and S. P. Chen, RSC Adv., 2016, 6, 67308-67314.

37 N. Al-Zaqri, A. Alsalme, S. F. Adil, A. Alsaleh, S. G. Alshammari, S. I. Alresayes, R. Alotaibi, M. Al-Kinany and M. R. H. Siddiqui, J. Saudi Chem. Soc., 2017, 21, 965-973.
38 S. Dharne and V. V. Bokade, J. Nat. Gas Chem., 2011, 20, 1824.

39 S. H. Teo, U. Rashid and Y. H. Taufiq-Yap, $R S C A d v ., 2014,4$, 48836-48847.

40 H. V. Lee, R. Yunus, J. C. Juan and Y. H. Taufiq-Yap, Fuel Process. Technol., 2011, 92, 2420.

41 H. T. Wu, J. H. Zhang, Y. P. Liu, J. L. Zheng and Q. Wei, Fuel Process. Technol., 2014, 119, 114-120.

42 Y. H. Taufiq-Yap, H. Lee, M. Hussein and R. Yunus, Biomass Bioenergy, 2011, 35, 827-834.

43 K. M. Parida and S. J. Mallick, J. Mol. Catal. A: Chem., 2007, 275, 77-83.

44 N. Kaur and A. Ali, Renewable Energy, 2015, 81, 421-431.

45 V. Brahmkhatri and A. Patel, Fuel, 2012, 102, 72-77.

46 M. Banchero and G. Gozzelino, Energies, 2018, 11, 1843.

47 W. Shi, B. He and J. Li, Bioresour. Technol., 2011, 102, 53895393.

48 H. L. Zhang, J. R. Gao, Z. D. Zhao, G. Z. Chen, T. Wu and F. He, Catal. Sci. Technol., 2016, 6, 5590-5598.

49 A. A. Costa, P. R. S. Braga, J. L. de Macedo, J. A. Dias and S. C. L. Dias, Microporous Mesoporous Mater., 2012, 147, 142-148.

50 F. J. Liu, W. Li, Q. Sun, L. F. Zhu, X. J. Meng, Y. H. Guo and F. S. Xiao, ChemSusChem, 2011, 4, 1059-1062.

51 M. L. Grecea, A. C. Dimian, S. Tanase, V. Subbiah and G. Rothenberg, Catal. Sci. Technol., 2012, 2, 1500-1506.

52 M. A. R. Melo Júnior, C. E. Albuquerque, J. S. A. Carneiro, C. Dariva, M. Fortuny, A. F. Santos, S. M. S. Egues and A. L. D. Ramos, Ind. Eng. Chem. Res., 2010, 49, 12135-12139. 53 S. Gopinath, P. Sahaya Murphin Kumar, K. A. Yasar Arafath, K. V. Thiruvengadaravi, S. Sivanesan and P. Baskaralingam, Fuel, 2017, 203, 488-500. 